\title{
Bioactive Components and Antioxidant and Antibacterial Activities of Different Varieties of Honey: A Screening Prior to Clinical Application
}

\author{
Patricia Combarros-Fuertes, ${ }^{\dagger}$ Leticia M. Estevinho, ${ }^{\ddagger}$ Luis G. Dias, ${ }^{\ddagger}$ José M. Castro, ${ }^{\S}$
}

Francisco A. Tomás-Barberán, ${ }^{\circledR}$ () M. Eugenia Tornadijo, ${ }^{\dagger}$ and José M. Fresno-Baro* ${ }^{*}{ }^{\dagger}$

†Department of Food Hygiene and Technology, Faculty of Veterinary Science, University of León, C/Profesor Pedro Cármenes s/n,
Campus de Vegazana, 24007 León, Spain
${ }^{\ddagger}$ CIMO, Mountain Research Center, Polytechnic Institute of Bragança, Campus Santa Apolónia, 5301-855 Bragança, Portugal
${ }^{\S}$ Departmentof Molecular Biology, Faculty of Biological and Environmental Sciences, University of León, Campus de Vegazana, s/n,
24007 León, Spain
${ }^{\|}$Research Group on Quality, Safety and Bioactivity of Plant Foods, CEBAS-CSIC. P.O. Box 164, 30100 Espinardo, Murcia, Spain

Supporting Information

ABSTRACT: This study assessed 16 different honey samples in order to select the best one for therapeutic purposes. First, a study of honey's main bioactive compounds was carried out. Then phenolic profiles were determined and specific compounds quantified using a HPLC system coupled to a mass spectrometer. Then, antioxidant activity, by three in vitro methods, and antibacterial activity against reference strains and clinical isolates were evaluated. Great variability among samples was observed regarding ascorbic acid (between $0.34 \pm 0.00$ and $75.8 \pm 0.41 \mathrm{mg} / 100 \mathrm{~g}$ honey; $p<0.001$ ), total phenolic compounds (between $23.1 \pm 0.83$ and $158 \pm 5.37 \mathrm{mg} / 100 \mathrm{~g}$ honey; $p<0.001$ ), and total flavonoid contents (between $1.65 \pm 0.11$ and 5.93 $\pm 0.21 \mathrm{mg} / 100 \mathrm{~g}$ honey; $p<0.001$ ). Forty-nine different phenolic compounds were detected, but only 46 of them were quantified by HPLC. The concentration of phenolic compounds and the phenolic profiles varied widely among samples (between $1.06 \pm 0.04$ and $18.6 \pm 0.73 \mathrm{mg} / 100 \mathrm{~g}$ honey; $p<0.001$ ). Antioxidant activity also varied significantly among the samples. All honey varieties exhibited antibacterial activity against both reference and clinical strains (effective concentrations ranged between 0.05 and $0.40 \mathrm{~g} / \mathrm{mL}$ depending on the honey sample and bacteria tested). Overall, samples with better combinations of bioactive properties were avocado and chestnut honeys.

KEYWORDS: honey, bioactive compounds, antioxidant activity, antibacterial activity, phenolic profiles

\section{INTRODUCTION}

Honey has been used as a medicinal remedy throughout the history of the human race: from ancient Egypt and the Classic civilizations (Greeks and Romans), who used honey in medicinal formulas, cosmetics, and perfumery or as embalming substance, to the Arab people of the Middle Ages, for whom honey was the basis of their pharmacy, as reflected in the Quran. ${ }^{1}$ In modern medicine, with the advent of antibiotics and other drugs, the use of honey was abandoned, mainly due to the absence of scientific studies. However, in recent decades, several investigations have demonstrated the bioactive properties by which honey was empirically used. ${ }^{2}$

The miscellaneous composition of honey is responsible for the attributable numerous bioactive properties. Certain enzymes such as glucose oxidase and catalase, ascorbic acid, carotenoids, and melanoidins (Maillard reaction products) as well as phenolic acids and flavonoids are related to its antioxidant activity. ${ }^{3}$ Antibacterial properties are associated with intrinsic characteristics such as high osmolarity and acidity and compounds such as hydrogen peroxide $\left(\mathrm{H}_{2} \mathrm{O}_{2}\right)$, methyl syringate and methylglyoxal, defensin-1, nitric oxide metabolites, and phenolic acids and flavonoids. ${ }^{4-7}$ In addition, honey may increase lymphocytic and phagocytic activity and likewise antibody production. ${ }^{5}$

The majority of recent studies investigating the bioactivity and the action mechanisms of honey have focused on wellcharacterized, standardized active manuka honey $(\mathrm{MkH})$. $\mathrm{MkH}$ is produced from the nectar of different Leptospermum species, native to New Zealand and Australia. Its greater activity is related to non-peroxide activity, due to the presence of an abundant suite of phenolic compounds, such as methyl glyoxal, methyl syringate, and leptosin, that distinguish them from other types of honey. ${ }^{2,8,9}$ However, in recent years, more and more studies are demonstrating the bioactive properties of other varieties of honey different from $\mathrm{MkH}$.

Unfortunately, honey composition is rather variable, depending primarily on botanical origin, ${ }^{10}$ conditioning its bioactive potential and hampering its further application for clinical purposes. ${ }^{3,411}$ This fact highlights the importance of selecting an adequate variety of honey to carry out clinical

Received: October 11, 2018

Revised: December 20, 2018

Accepted: December 21, 2018

Published: December 21, 2018 
assays, ${ }^{7}$ which means a previous screening is necessary in order not only to quantify but also to determine profiles of bioactive substances, especially phenolic compounds; thus, the variation in these profiles might be responsible for the widely varying medical abilities of honey.

The working hypothesis of this study is whether distinct varieties of Spanish honey exhibit rather variable composition, especially regarding bioactive compounds, and in consequence significantly different therapeutic potential. In order to validate the veracity of this assumption, the aims of the study were (i) to quantify bioactive compounds in different honey samples (including a $\mathrm{MkH}$ sample as control), (ii) to identify and quantify individual phenolic compounds as major bioactive components present in honey, (iii) to determine the antioxidant activity of honey samples, and (iv) to determine their antibacterial activity against reference strains and clinical isolates. The overall goal was to compare different types of Spanish honey to select one that shows the best properties for potential therapeutic applications.

\section{MATERIALS AND METHODS}

Chemicals. Acetonitrile, acetic acid, formic acid, methanol, sodium carbonate, hydrochloric acid, and metaphosphoric acid were supplied by VWR Chemicals-Prolabo (VWR International). 2,2Diphenyl-1-picrylhydrazyl ( $\left.\mathrm{DPPH}^{\bullet}\right)$, 2,6-dichloroindophenol, caffeic and gallic acids, flavonoid standards (rutin, quercetin, chrysin, and catechin), aluminum chloride, ferric chloride, and potassium ferricyanide were supplied by Sigma (St. Louis, MO, USA). All other chemicals were obtained from Merck (Darmstadt, Germany). All solvents were of analytical grade purity except for methanol, formic acid, and acetonitrile used in the identification and quantification of individual polyphenols, which were HPLC grade. Water was treated in a Milli-Q water purification system (Millipore, Molsheim, France).

Honey Samples. Fifteen samples of Spanish honey under quality brands (protected designation of origin Miel de Granada and Miel de La Alcarria, protected geographical indication Miel de Galicia and organic honey) with different botanical and geographical origins, as well as an MkH sample (MGO 550+; Manuka Health, Auckland, New Zealand), as a control sample, were used. Spanish honey samples, collected in two consecutive harvests, were previously characterized. ${ }^{12}$ Table 1 summarizes the information related to botanical and geographical origin of honey samples, as well as the harvest year.

The samples were stored under dark conditions and refrigeration until analysis (few months after harvesting). They were homogenized by agitation before each determination.

Bioactive Compound Quantification. Vitamin C Content. Ascorbic acid (AA) content was determined following the recommended AOAC Official Titrimetric Method 967.21 for ascorbic acid in vitamin preparations and juices. ${ }^{13} \mathrm{~A} 5 \mathrm{~g}$ portion of each sample was diluted in $5 \mathrm{~mL}$ of metaphosphoric acid acetic acid solution. The mixture was titrated with 2,6- dichloroindophenol dye solution. Vitamin C content was expressed in milligrams of ascorbic acid equivalents (AAE) per $100 \mathrm{~g}$ of honey.

Total Phenolic Content. Total phenolic content (TPC) was quantified by the Folin-Ciocalteu method according to Silici, Sagdic, and Ekici. ${ }^{14}$ Absorbance was measured at $765 \mathrm{~nm}$ after $90 \mathrm{~min}$ of incubation at room temperature (UV-vis spectrophotometer; VWR UV-3100 PC). TPC was determined using a standard curve $(y=$ $\left.32.08 x+0.012 ; R^{2}=0.9996\right)$ of gallic acid $(0-0.03 \mathrm{mg} / \mathrm{mL})$. The results were expressed as milligrams of gallic acid equivalents per 100 $\mathrm{g}$ of honey.

Total Flavonoid Content. Total flavonoid content (TFC) was determined using the protocol described by Alvarez-Suarez et al. ${ }^{3}$ A cadmium chloride solution was replaced by an aluminum chloride $\left(\mathrm{AlCl}_{3}\right)$ solution $(10 \% \mathrm{w} / \mathrm{v})$. Absorbance was measured immediately against the blank at $510 \mathrm{~nm}$ (UV-vis spectrophotometer; VWR UV-
Table 1. Confirmed Botanical Origin, Year of Harvest, Quality Brand, and Geographical Origin of Spanish Honey Samples

\begin{tabular}{|c|c|c|c|c|}
\hline $\begin{array}{c}\text { sample } \\
\text { identification }\end{array}$ & botanical origin & $\begin{array}{c}\text { harvest } \\
\text { year }\end{array}$ & quality brand & $\begin{array}{l}\text { geographical } \\
\text { origin }\end{array}$ \\
\hline $\mathrm{H} 1$ & multifloral & 2010 & $\begin{array}{l}\mathrm{PDO}^{a} \text { Miel de } \\
\text { Granada }\end{array}$ & $\begin{array}{l}\text { Province of } \\
\text { Granada }\end{array}$ \\
\hline H1a & $\begin{array}{l}\text { avocado (Persea } \\
\text { americana) }\end{array}$ & 2011 & $\begin{array}{l}\text { PDO Miel de } \\
\text { Granada }\end{array}$ & $\begin{array}{c}\text { Province of } \\
\text { Granada }\end{array}$ \\
\hline $\mathrm{H} 2$ & $\begin{array}{c}\text { chestnut (Castanea } \\
\text { sativa) }\end{array}$ & 2010 & $\begin{array}{l}\text { PDO Miel de } \\
\text { Granada }\end{array}$ & $\begin{array}{l}\text { Province of } \\
\text { Granada }\end{array}$ \\
\hline $\mathrm{H} 2 \mathrm{a}$ & $\begin{array}{l}\text { chestnut (Castanea } \\
\text { sativa) }\end{array}$ & 2011 & $\begin{array}{l}\text { PDO Miel de } \\
\text { Granada }\end{array}$ & $\begin{array}{l}\text { Province of } \\
\text { Granada }\end{array}$ \\
\hline $\mathrm{H} 3$ & multifloral & 2010 & $\begin{array}{l}\text { PDO Miel de } \\
\text { La Alcarria }\end{array}$ & $\begin{array}{l}\text { Province of } \\
\text { Cuenca }\end{array}$ \\
\hline $\mathrm{H} 4$ & $\begin{array}{l}\text { rosemary (Rosmari- } \\
\text { nus officinalis) }\end{array}$ & 2010 & $\begin{array}{l}\text { PDO Miel de } \\
\text { La Alcarria }\end{array}$ & $\begin{array}{l}\text { Province of } \\
\text { Cuenca }\end{array}$ \\
\hline $\mathrm{H} 4 \mathrm{a}$ & multifloral & 2011 & $\begin{array}{l}\text { PDO Miel de } \\
\text { La Alcarria }\end{array}$ & $\begin{array}{l}\text { Province of } \\
\text { Cuenca }\end{array}$ \\
\hline H5 & multifloral & 2010 & $\begin{array}{l}\mathrm{PGI}^{a} \text { Miel de } \\
\text { Galicia }\end{array}$ & $\begin{array}{l}\text { Province of } \\
\text { Pontevedra }\end{array}$ \\
\hline $\mathrm{H} 5 \mathrm{a}$ & multifloral & 2011 & $\begin{array}{l}\text { PGI Miel de } \\
\text { Galicia }\end{array}$ & $\begin{array}{l}\text { Province of } \\
\text { Pontevedra }\end{array}$ \\
\hline H6 & $\begin{array}{l}\text { eucalyptus (Euca- } \\
\text { lyptus sp.) }\end{array}$ & 2010 & $\begin{array}{l}\text { PGI Miel de } \\
\text { Galicia }\end{array}$ & $\begin{array}{l}\text { Province of } \\
\text { Pontevedra }\end{array}$ \\
\hline H6a & $\begin{array}{l}\text { eucalyptus (Euca- } \\
\text { lyptus sp.) }\end{array}$ & 2011 & $\begin{array}{l}\text { PGI Miel de } \\
\text { Galicia }\end{array}$ & $\begin{array}{l}\text { Province of } \\
\text { Pontevedra }\end{array}$ \\
\hline $\mathrm{H} 7$ & multifloral & 2010 & $\begin{array}{l}\text { certified organ- } \\
\text { ic honey }\end{array}$ & $\begin{array}{l}\text { Province of } \\
\text { León }\end{array}$ \\
\hline $\mathrm{H} 7 \mathrm{a}$ & thyme (Thymus sp.) & 2011 & $\begin{array}{l}\text { certified organ- } \\
\text { ic honey }\end{array}$ & $\begin{array}{l}\text { Province of } \\
\text { León }\end{array}$ \\
\hline $\mathrm{H} 8$ & $\begin{array}{l}\text { chestnut (Castanea } \\
\text { sativa) }\end{array}$ & 2010 & $\begin{array}{l}\text { certified organ- } \\
\text { ic honey }\end{array}$ & $\begin{array}{l}\text { Province of } \\
\text { León }\end{array}$ \\
\hline H8a & multifloral & 2011 & $\begin{array}{l}\text { certified organ- } \\
\text { ic honey }\end{array}$ & $\begin{array}{l}\text { Province of } \\
\text { León }\end{array}$ \\
\hline
\end{tabular}

${ }^{a} \mathrm{PDO}$, protected designation of origin; PGI, protected geographical indication.

3100 PC). TFC was determined using a standard curve $(y=10.99 x+$ $\left.0.0052 ; R^{2}=0.9997\right)$ of $(+)$-catechin $(0-0.03 \mathrm{mg} / \mathrm{mL})$. The results were expressed as milligrams of catechin equivalents per $100 \mathrm{~g}$ of honey.

Identification and Quantification of Individual Polyphenols. The identification and quantification of phenolic compounds were carried out following the protocol described by Truchado, Ferreres, and Tomás-Barberán ${ }^{15}$ with slight modifications.

Polyphenolic Extract Preparation. Honey samples (20 g) were fully dissolved in 8 parts of acidified deionized water (adjusted to $\mathrm{pH}$ 2 with $\mathrm{HCl}$ ). The solutions were centrifuged at $5000 \mathrm{rpm}$ for $10 \mathrm{~min}$ (Eppendorf 5804R), and the supernatant was applied to a Sep-Pak Classic $\mathrm{C}_{18}$ cartridge (Waters, Medford, MA, USA) with a dropwise flow rate to ensure an efficient adsorption of the phenolic compounds. Phenolic content was eluted with HPLC grade methanol $(2 \mathrm{~mL})$. This methanolic extract was filtered through a $0.45 \mu \mathrm{m}$ filter (Waters) and stored at $-20{ }^{\circ} \mathrm{C}$ until subsequent analysis by HPLC.

Identification and Quantification of Polyphenolic Compounds. HPLC analyses were performed using an Agilent 1100 HPLC system equipped with a photodiode-array UV-vis detector and an ion-trap mass spectrometer detector in series (Agilent Technologies, Waldbronn, Germany). Chromatographic separation was carried out on a reverse phase Poroshell120 $\mathrm{C}_{18}$ column $(250 \times 3.0 \mathrm{~mm}$ and 5 $\mu \mathrm{m}$ particle size) (Agilent Technologies) using water with $1 \%$ of formic acid (A) and acetonitrile (B) as mobile phases. The gradient profile was as follows: $0-20 \mathrm{~min}, 5-30 \% \mathrm{~B} ; 20-40 \mathrm{~min}, 30-70 \% \mathrm{~B}$; $40-45 \mathrm{~min}, 70-95 \% \mathrm{~B}$; $46-48 \mathrm{~min}, 95-5 \% \mathrm{~B}$; maintained at $5 \%$ for $55 \mathrm{~min}$. All analyses were carried out at room temperature, with an injected volume of $20 \mu \mathrm{L}$ and a flow rate of $1 \mathrm{~mL} / \mathrm{min}$. UV spectra were recorded from 210 to $600 \mathrm{~nm}$, whereas chromatograms were monitored at 280, 320, 340, and $360 \mathrm{~nm}$ wavelengths. 
Table 2. Calibration Parameters for Phenolic Acids and Flavonoids Used As Standards (mg/mL) and Compound Class To Be Quantified by Each Standard

\begin{tabular}{|c|c|c|c|c|c|c|}
\hline compound & linearity range & equation & $R^{2}$ & $\mathrm{LOD}^{a}$ & $\mathrm{LOQ}^{a}$ & group to be quantified \\
\hline gallic acid & $0.01-0.30$ & $y=49.39 x$ & 0.999 & 0.02 & 0.05 & hydroxybenzoic acids \\
\hline caffeic acid & $0.002-0.20$ & $y=146.82 x$ & 0.999 & 0.01 & 0.03 & hydroxycinammic acids \\
\hline quercetin & $0.002-0.20$ & $y=62.66 x$ & 0.999 & 0.01 & 0.04 & flavonols \\
\hline naringenin & $0.01-0.20$ & $y=50.84 x$ & 0.999 & 0.01 & 0.03 & flavanonols and flavanones \\
\hline chrysin & $0.01-0.30$ & $y=43.01 x$ & 0.999 & 0.01 & 0.03 & flavones \\
\hline rutin & $0.01-0.30$ & $y=69.31 x$ & 0.999 & 0.01 & 0.03 & flavonol glycosides \\
\hline
\end{tabular}

${ }^{a} \mathrm{LOD}$, limit of detection in $\mathrm{mg} / \mathrm{mL}$; LOQ, limit of quantification in $\mathrm{mg} / \mathrm{mL}$.

Table 3. Ascorbic Acid, Total Phenolic Compounds, Total Flavonoids and Antioxidant Activity of Honey Samples (Mean SD; $n=3)^{a}$

\begin{tabular}{|c|c|c|c|c|c|c|}
\hline \multirow[b]{2}{*}{ honey sample } & \multicolumn{3}{|c|}{ bioactive compounds } & \multicolumn{3}{|c|}{ antioxidant activity } \\
\hline & $\mathrm{AAE}^{b}$ & $\mathrm{TPC}^{c}$ & $\mathrm{TFC}^{d}$ & radical scavenging activity ${ }^{e}$ & reducing potential $f$ & $\beta$-carotene bleaching inhibition \\
\hline H1 & $9.11 \pm 0.61^{b}$ & $158 \pm 5.37^{\mathrm{a}}$ & $5.93 \pm 0.21^{\mathrm{a}}$ & $9.25 \pm 0.32^{\mathrm{mn}}$ & $26.3 \pm 1.29^{\mathrm{m}}$ & $32.9 \pm 1.47^{\mathrm{ij}}$ \\
\hline H1a & $5.95 \pm 0.32^{\mathrm{cd}}$ & $117 \pm 2.74^{\mathrm{d}}$ & $3.30 \pm 0.08^{\mathrm{bc}}$ & $13.8 \pm 0.07^{\mathrm{kl}}$ & $30.3 \pm 0.04^{\mathrm{lm}}$ & $56.9 \pm 0.99^{g}$ \\
\hline $\mathrm{H} 2$ & $3.64 \pm 0.30^{f}$ & $102 \pm 1.53^{\mathrm{ef}}$ & $2.29 \pm 0.14^{\text {cde }}$ & $23.0 \pm 0.38^{\mathrm{h}}$ & $55.3 \pm 1.49^{\mathrm{i}}$ & $66.8 \pm 1.76^{\mathrm{ef}}$ \\
\hline $\mathrm{H} 2 \mathrm{a}$ & $6.62 \pm 0.05^{\mathrm{bc}}$ & $118 \pm 3.50^{\mathrm{cd}}$ & $5.85 \pm 0.21^{\mathrm{a}}$ & $9.83 \pm 0.07^{\mathrm{lm}}$ & $43.0 \pm 1.30^{j}$ & $92.6 \pm 0.58^{\mathrm{ab}}$ \\
\hline H3 & $2.41 \pm 0.18^{\mathrm{fg}}$ & $67.9 \pm 1.48^{\text {gh }}$ & $4.06 \pm 0.04^{\mathrm{ab}}$ & $38.0 \pm 0.45^{\mathrm{ef}}$ & $34.7 \pm 0.70^{\mathrm{jk}}$ & $58.4 \pm 1.40^{\mathrm{fg}}$ \\
\hline H4 & $4.51 \pm 0.00^{\mathrm{d}}$ & $23.1 \pm 0.83^{1}$ & $2.17 \pm 0.11^{\operatorname{defg}}$ & $202 \pm 5.53^{\mathrm{a}}$ & $215 \pm 1.81^{\mathrm{a}}$ & $28.3 \pm 1.09^{\mathrm{jk}}$ \\
\hline $\mathrm{H} 4 \mathrm{a}$ & $0.34 \pm 0.00^{\mathrm{k}}$ & $27.7 \pm 1.45^{\mathrm{kl}}$ & $2.02 \pm 0.19^{\mathrm{efgh}}$ & $119 \pm 0.02^{\mathrm{ab}}$ & $157 \pm 1.47^{\mathrm{ab}}$ & $38.0 \pm 0.44^{\mathrm{hi}}$ \\
\hline H5 & $1.35 \pm 0.11^{\mathrm{hi}}$ & $67.5 \pm 2.65^{\text {gh }}$ & $1.95 \pm 0.15^{\mathrm{fgh}}$ & $28.9 \pm 0.43^{\mathrm{fg}}$ & $93.5 \pm 0.35^{\mathrm{ef}}$ & $92.9 \pm 0.52^{\mathrm{a}}$ \\
\hline H5a & $0.88 \pm 0.00^{\mathrm{ij}}$ & $56.6 \pm 0.29^{\mathrm{hi}}$ & $1.89 \pm 0.14^{\mathrm{gh}}$ & $28.2 \pm 1.24^{\mathrm{g}}$ & $82.4 \pm 0.95^{\mathrm{fg}}$ & $92.4 \pm 0.28^{\mathrm{ab}}$ \\
\hline H6 & $0.34 \pm 0.00^{k}$ & $50.6 \pm 1.64^{j}$ & $1.65 \pm 0.11^{\mathrm{h}}$ & $55.9 \pm 0.35^{\mathrm{cd}}$ & $111 \pm 1.02^{\mathrm{de}}$ & $71.8 \pm 1.13^{\mathrm{de}}$ \\
\hline H6a & $0.35 \pm 0.00^{\mathrm{k}}$ & $50.5 \pm 1.69^{j}$ & $1.83 \pm 0.22^{\mathrm{efgh}}$ & $74.1 \pm 0.84^{\mathrm{bc}}$ & $118 \pm 0.16^{\mathrm{cd}}$ & $82.1 \pm 0.17^{\mathrm{bc}}$ \\
\hline H7 & $0.34 \pm 0.00^{k}$ & $51.3 \pm 2.80^{\mathrm{ij}}$ & $2.25 \pm 0.11^{\text {cdef }}$ & $54.0 \pm 0.81^{\mathrm{de}}$ & $147 \pm 4.51^{\mathrm{bc}}$ & $68.1 \pm 0.86^{\mathrm{ef}}$ \\
\hline H7a & $75.9 \pm 0.41^{\mathrm{a}}$ & $136 \pm 2.50^{\mathrm{bc}}$ & $2.06 \pm 0.22^{\mathrm{abc}}$ & $5.46 \pm 0.05^{\mathrm{n}}$ & $54.1 \pm 0.64^{\mathrm{i}}$ & $-1.34 \pm 0.10^{\mathrm{k}}$ \\
\hline H8 & $3.61 \pm 0.18^{\mathrm{ef}}$ & $142 \pm 4.70^{\mathrm{ab}}$ & $2.97 \pm 0.19^{\mathrm{bcd}}$ & $21.6 \pm 0.33^{\mathrm{ij}}$ & $72.0 \pm 0.78^{g h}$ & $31.9 \pm 1.51^{\mathrm{ij}}$ \\
\hline H8a & $4.22 \pm 0.32^{\mathrm{de}}$ & $114 \pm 4.23^{\mathrm{de}}$ & $3.87 \pm 0.04^{\mathrm{efgh}}$ & $15.1 \pm 0.25^{\mathrm{jk}}$ & $63.7 \pm 0.16^{\mathrm{h}}$ & $78.4 \pm 0.84^{\mathrm{cd}}$ \\
\hline MkH & $2.19 \pm 0.13^{\mathrm{gh}}$ & $101 \pm 1.92^{\mathrm{fg}}$ & $4.76 \pm 0.26^{\mathrm{ab}}$ & $22.6 \pm 0.50^{\mathrm{hi}}$ & $32.8 \pm 0.33^{\mathrm{kl}}$ & $43.9 \pm 0.91^{\text {gh }}$ \\
\hline
\end{tabular}

${ }^{a}$ In each column different letters mean significant differences $(p<0.05) .{ }^{b} \mathrm{AAE}$ : ascorbic acid equivalents $\left(\mathrm{mg}\right.$ per $100 \mathrm{~g}$ of honey). ${ }^{c}$ TPC: total phenolic content equivalents of gallic acid (mg per $100 \mathrm{~g}$ of honey). ${ }^{d}$ TFC: total flavonoids content equivalents of catechin (mg per $100 \mathrm{~g}$ of honey). ${ }^{e} \mathrm{EC}_{50}$ : extract concentration $(\mathrm{mg} / \mathrm{mL})$ providing $50 \%$ of radical scavenging activity. ${ }^{f} \mathrm{EC}_{50}$ : extract concentration $(\mathrm{mg} / \mathrm{mL})$ providing 0.5 of absorbance. ${ }^{g}$ Antioxidant activity: percentage of inhibition of $\beta$-carotene oxidation.

The HPLC system was coupled in series to an Esquire 1100 iontrap mass spectrometer (IT) equipped with an electrospray ionization interface (ESI) (Bruker, Bremen, Germany) in negative mode. Nitrogen was used as a drying gas with a flow of $9 \mathrm{~L} / \mathrm{min}$ and temperature of $350{ }^{\circ} \mathrm{C}$ and nebulizing gas at a pressure of $40 \mathrm{psi}$. The capillary voltage was set at 3500 V. Mass scan (MS) and daughter (MS-MS) spectra were recorded in the range of $\mathrm{m} / z 100-1500$ with a control mass of $m / z 700$. The analyses were performed in duplicate.

Honey phenolic acids and flavonoids were identified according to their molecular weight (mass spectra), characteristic UV spectra, MS/ MS fragmentations, and the wide information previously reported in the literature. Hydroxybenzoic acids were quantified using UV detection at $280 \mathrm{~nm}$ with the calibration curve obtained for gallic acid, hydroxycinnamic acids at $320 \mathrm{~nm}$ with the calibration curve obtained for caffeic acid, flavonols at $360 \mathrm{~nm}$ with the calibration curve of quercetin, flavanones at $280 \mathrm{~nm}$ with the calibration curve of naringenin, and flavones and flavonol glycosides at $340 \mathrm{~nm}$ with the calibration curves of chrysin and rutin, respectively. Calibration parameters are shown in Table 2.

Antioxidant Activity. Radical Scavenging Activity Assay. The radical scavenging activity (RSA) of honey samples was evaluated using the DPPH radical scavenging assay following the protocol described by Ferreira, Aires, Barreira, and Estevinho. ${ }^{16}$ The concentration of water honey solutions tested ranged between 0 and $0.67 \mathrm{~g} / \mathrm{mL}$. Radical scavenging activity was calculated as a percentage of DPPH discoloration using the equation \% RSA = $\left[\left(A_{\mathrm{DPPH}}-A_{\mathrm{S}}\right) / A_{\mathrm{DPPH}}\right] \times 100$. The extract concentration providing $50 \%$ of radical scavenging activity $\left(\mathrm{EC}_{50}\right)$ was calculated by interpolation from the graph of RSA percentage against extract concentration.

Reducing Potential Assay. The ferric reduction power (RP) was evaluated using the protocol described by Ferreira, Aires, Barreira, and Estevinho. ${ }^{16}$ The concentration of water honey solutions tested ranged between 0 and $0.11 \mathrm{~g} / \mathrm{mL}$. The extract concentration providing 0.5 of absorbance $\left(\mathrm{EC}_{50}\right)$ was calculated by interpolation from the graph of absorbance at $700 \mathrm{~nm}$ against extract concentration.

Inhibition of $\beta$-Carotene Bleaching Assay. The inhibition of $\beta$ carotene bleaching by honey samples was evaluated following the protocol described by Guerrini et al. ${ }^{17}$ with slight modifications. A 4 $\mathrm{mL}$ portion of $\beta$-carotene in chloroform solution $(0.2 \mathrm{mg} / \mathrm{mL})$ was pipetted into a round-bottom flask containing $80 \mu \mathrm{L}$ of linoleic acid and $800 \mu \mathrm{L}$ of Tween 40 as emulsifier. The mixture was shaken, and chloroform was removed at $40{ }^{\circ} \mathrm{C}$ under vacuum. A $200 \mathrm{~mL}$ portion of distilled water, previously swamped in $\mathrm{O}_{2}$, was added to the flask, which was vigorously shaken. Aliquots of $4.8 \mathrm{~mL}$ of this emulsion were transferred into different test tubes containing $0.2 \mathrm{~mL}$ of 300 $\mathrm{mg} / \mathrm{mL}$ water-honey solutions. The tubes were shaken and incubated in darkness at $55{ }^{\circ} \mathrm{C}$. The absorbance was measured at $470 \mathrm{~nm}$ (VWR UV-3100 PC) at the moment of emulsion addition and after $120 \mathrm{~min}$. An emulsion without $\beta$-carotene was used as a control. The antioxidant activity (AA) expressed as a percentage of inhibition of $\beta$-carotene oxidation was calculated using the equation $\mathrm{AA}=\left[100\left(\mathrm{DR}_{\mathrm{C}}-\mathrm{DR}_{\mathrm{S}}\right)\right] / \mathrm{DR}_{\mathrm{C}}$; where $\mathrm{DR}_{\mathrm{C}}=\ln (a / b) / 120$ is the percentage of degradation of $\beta$-carotene in the control and $\mathrm{DR}_{\mathrm{S}}=$ $\ln (a / b) / 120$ is the percentage degradation of $\beta$-carotene in honey 
Table 4. Peak Numbers, Target Compounds, Average Expected Retention Times $\left(R_{t}\right)$, and UV and MS Spectra of the Different Phenolic Compounds Identified in Honey Samples

\begin{tabular}{|c|c|c|c|c|c|}
\hline peak no. & compound name & $R_{\mathrm{t}}(\min )$ & $\mathrm{UV}_{\max }(\mathrm{nm})$ & {$[\mathrm{M}-\mathrm{H}]^{-}(m / z)$} & $-\operatorname{MS}^{\mathrm{n}}(m / z)$ \\
\hline 1 & UI 1 & 8.45 & $306 \mathrm{sh}, 316,328 \mathrm{sh}$ & 188 & 144 \\
\hline 2 & UI 2 & 10.29 & 318 sh, 330 & 188 & 144 \\
\hline 3 & kynurenic acid & 10.77 & $308,332,335 \mathrm{sh}, 340 \mathrm{sh}$ & 188 & 144 \\
\hline 4 & caffeic acid & 11.59 & 238,296 sh, 322 & 179 & 161,135 \\
\hline 5 & leptosperin & 11.84 & $266,296 \mathrm{sh}$ & 581 & 323,211 \\
\hline 6 & quercetin-3-O-hex $(1 \rightarrow 2)$ hex $^{a}$ & 13.92 & 259,265 sh, 299 sh, 355 & 625 & 445,301 \\
\hline 7 & 8-methoxykaempferol-3-O- hex $(1 \rightarrow 2)$ hex ${ }^{a}$ & 14.87 & & 639 & $624,459,315$ \\
\hline 8 & kaempferol-3-O-hex $(1 \rightarrow 2)$ hex $^{a}$ & 15.39 & 265,296 sh, 349 & 609 & $447,429,285$ \\
\hline 9 & trans-cinnamic acid & 15.75 & 276 & 147 & 119,103 \\
\hline 10 & 8-O-methoxykaempferol-3-O-neoh ${ }^{a}$ & 15.97 & $310 \mathrm{sh}, 324,362 \mathrm{sh}$ & 623 & $608,459,315$ \\
\hline 11 & quercertin-3-O-rutinoside & 16.25 & 258,260 sh, 291 sh, 349 & 609 & 301 \\
\hline 12 & ellagic acid & 16.50 & 253,367 & 301 & $301,257,229$ \\
\hline 13 & kaempferol-3-O-neoh ${ }^{a}$ & 16.62 & 248,262 sh, 298 sh, 326 & 593 & 429,285 \\
\hline 14 & 4-methoxyphenyllactic acid & 16.70 & 274 & 195 & 177,149 \\
\hline 15 & UI 3 & 16.73 & $298 \mathrm{sh}, 309,319 \mathrm{sh}$ & 144 & 133 \\
\hline 16 & isorhamnetin-3-O-neoh $^{a}$ & 16.83 & & 623 & 459,315 \\
\hline 17 & Chlorogenic acid & 18.40 & 298,328 & 353 & 191,179 \\
\hline 18 & isorhamnetin-O-pentoside & 18.97 & 253,346 & 447 & 315,300 \\
\hline 19 & rosmarinic acid & 20.23 & 294,329 & 359 & $329,286,234$ \\
\hline 20 & myricetin & 20.30 & $255,267 \mathrm{sh}, 301 \mathrm{sh}, 375$ & 317 & 179,151 \\
\hline 21 & tricetin & 21.07 & 248,267 sh, 302 sh, 351 & 301 & 151 \\
\hline 22 & methyl syringate & 21.30 & 274 & 211 & 181 \\
\hline 23 & quercetin-3-O-rham $^{a}$ & 21.63 & & 447 & 301 \\
\hline 24 & trans, trans-abscisic acid & 21.87 & 266 & 263 & 219,201 \\
\hline 25 & cis,trans-abscisic acid & 23.52 & 266 & 263 & 219,201 \\
\hline 26 & quercetin & 24.46 & 255,370 & 301 & $179,151,121$ \\
\hline 27 & naringenin 7-methyl ether & 25.25 & $288,320 \mathrm{sh}$ & 285 & $267,252,239$ \\
\hline 28 & pinobanksin-5-methyl ether & 25.31 & 286 & 285 & $267,252,239$ \\
\hline 29 & quercetin 3-methyl ether & 25.70 & 256,355 & 315 & $300,271,255$ \\
\hline 30 & $p$-coumaric acid isoprenil ester & 26.42 & 294,310 & 231 & 163,119 \\
\hline 31 & pinobanksin & 27.42 & 292 & 271 & $253,225,151$ \\
\hline 32 & kaempferol & 27.72 & 266,370 & 285 & $161,151,135$ \\
\hline 33 & isorhamnetin & 28.37 & 253,370 & 315 & $300,151,107$ \\
\hline 34 & kaempferol methyl ether & 28.79 & 265,352 & 299 & 284 \\
\hline 35 & kaempferide & 28.80 & 265,364 & 299 & $284,228,212,151,132$ \\
\hline 36 & quercetin 3,3-dimethyl ether & 29.43 & 253,355 & 329 & $314,299,285,271$ \\
\hline 37 & rhamnetin & 30.94 & 256,367 & 315 & $300,165,121$ \\
\hline 38 & quercetin 3,7-dimethyl ether & 32.00 & 256,355 & 329 & $314,299,285$ \\
\hline 39 & caffeic acid isoprenyl ester & 32.83 & 298,325 & 247 & 179,135 \\
\hline 40 & caffeic acid benzyl ester & 33.17 & 298,325 & 269 & $178,161,134$ \\
\hline 41 & chrysin & 33.31 & $268,314 \mathrm{sh}$ & 253 & $181,151,101$ \\
\hline 42 & pinocembrin & 33.57 & 289 & 255 & $213,211,151$ \\
\hline 43 & galangin & 34.03 & 265,360 & 269 & $269,241,151$ \\
\hline 44 & caffeic acid phenylethyl ester & 34.24 & 295,325 & 283 & 179,135 \\
\hline 45 & 6-methoxychrysin & 35.08 & $265,300 \mathrm{sh}, 346 \mathrm{sh}$ & 283 & $268,239,211$ \\
\hline 46 & galangin 5-methyl ether isomer & 35.11 & $266,302 \mathrm{sh}, 360$ & 283 & 268,239 \\
\hline 47 & caffeic acid cinnamyl ester & 36.05 & 295,324 & 295 & 178,134 \\
\hline 48 & pinobanksin-3-O-butyrate or isomer & 39.28 & 292 & 341 & 271,253 \\
\hline 49 & pinobanksin-3-O-pentenoate or isomer & 41.43 & 292 & 353 & 271,253 \\
\hline
\end{tabular}

samples: $a=$ absorbance at time $0 ; b=$ absorbance after $120 \mathrm{~min}$ of incubation.

Antibacterial Activity. Bacterial Strains, Drug Susceptibility, and Growth Conditions. Staphylococcus aureus, Streptococcus pyogenes, Escherichia coli, and Pseudomonas aeruginosa as the main bacteria isolated from the oropharynx of patients suffering from oral mucositis (University Assistance Complex of León, Spain), as well as strains of these species from the Spanish Type Culture Collection (S. aureus CECT 86, S. pyogenes CECT 985, E. coli CECT 515 and P. aeruginosa
CECT 110) were used. Clinical bacteria were identified using a MicroScan panel by Siemens (Camberley, U.K.).

The susceptibility of bacteria to different antibiotics was assessed by a plate microdilution method or a disk-plate diffusion method. Breakpoints were determined according to values defined by the Clinical and Laboratory Standards Institute. ${ }^{18}$ Clinical strains, excluding S. pyogenes, exhibited resistance to several antibiotics tested. S. aureus was a methicillin-resistant strain (MRSA), E. coli was a 


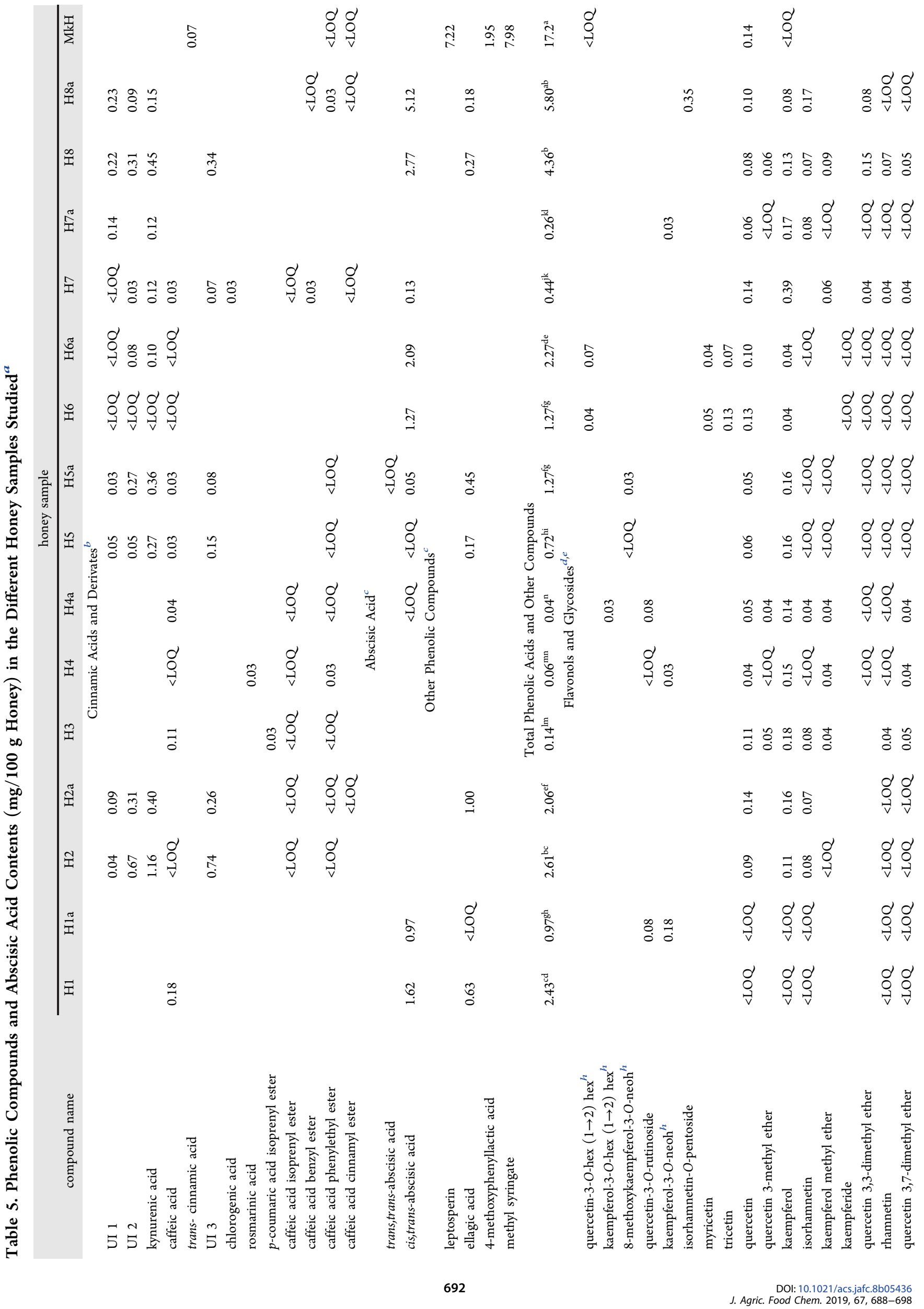




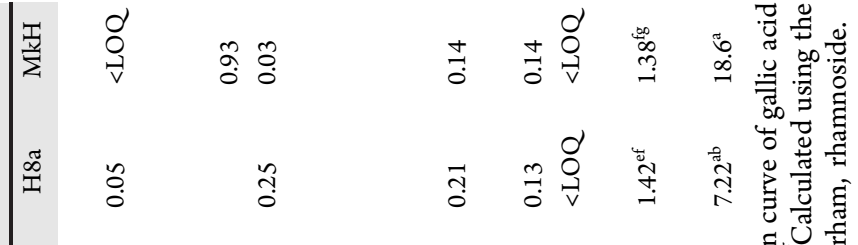

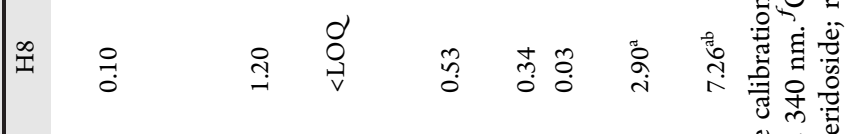

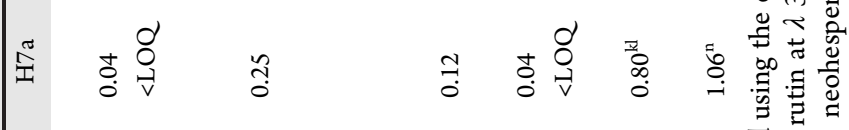

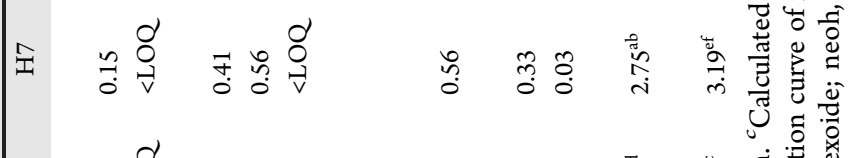

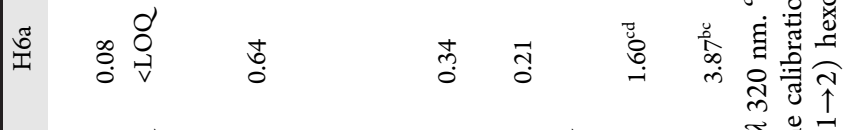

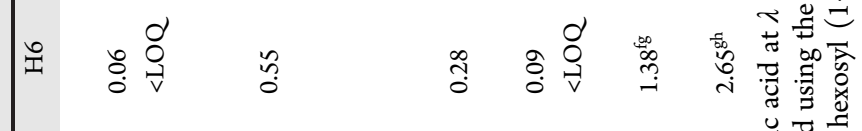

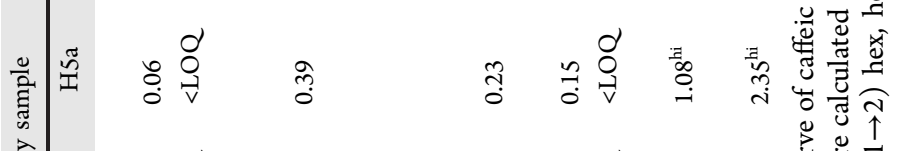

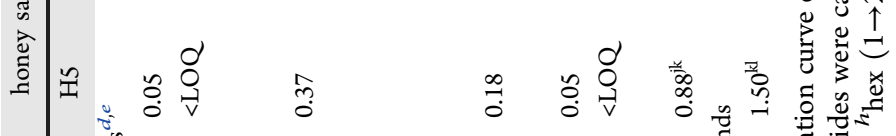

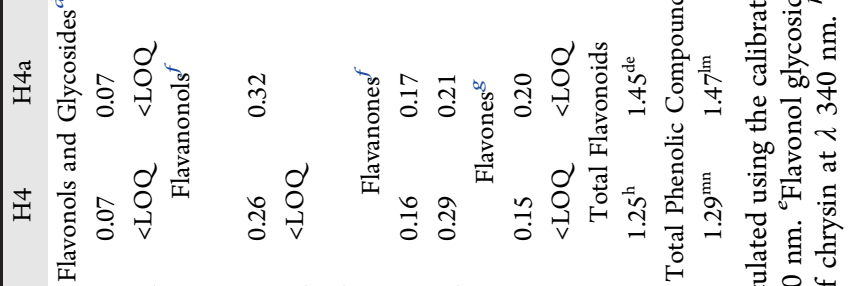

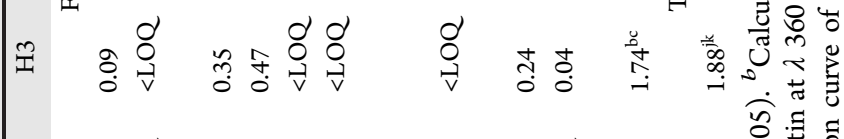

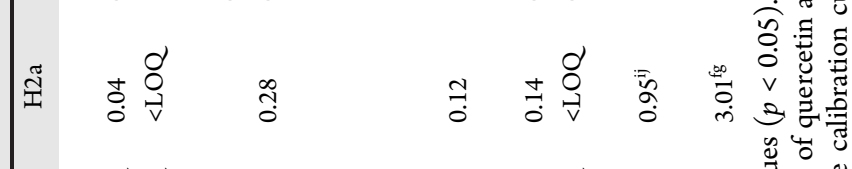

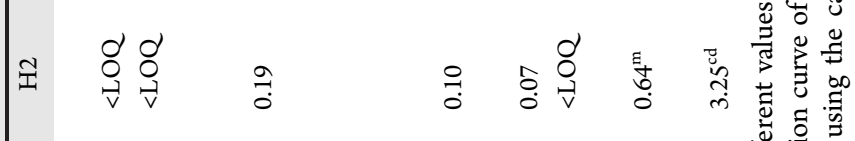

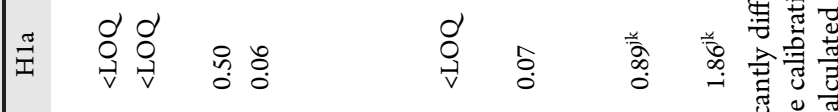

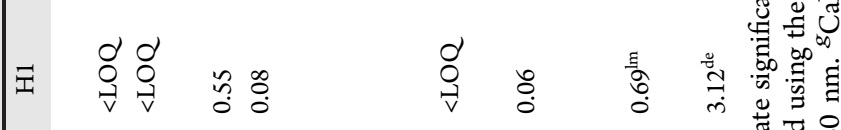

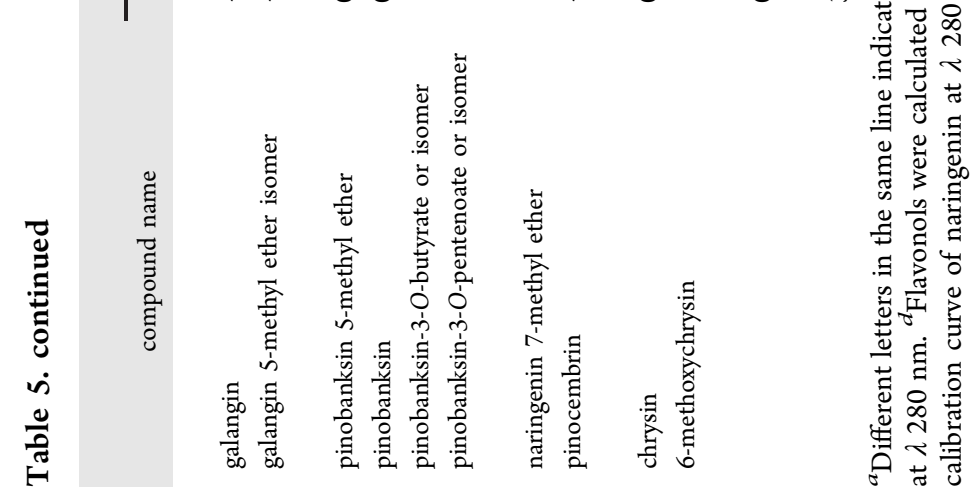


producer of $\beta$-lactamases, and $P$. aeruginosa showed resistance against 9 of 14 antibiotics tested (Supporting Information).

All bacteria were grown in Mueller Hinton broth $(\mathrm{MH}$; SigmaAldrich, St. Louis, MO, USA) at $37^{\circ} \mathrm{C}$ with shaking ( $\left.180 \mathrm{rpm}\right)$ until the exponential growth phase (JP Selecta, Barcelona, Spain). Prior to experiments, bacteria were subcultured twice in $\mathrm{MH}$ agar to ensure the purity of cultures.

Honey Susceptibility. The minimal inhibitory concentration (MIC) was determined according to the M07-A9 protocol. ${ }^{18}$ Honey concentrations between 400 and $6.25 \mathrm{mg} / \mathrm{mL}$ were tested. MIC values were defined after 24 and $48 \mathrm{~h}$ of incubation. MIC was the lowest concentration that prevented any discernible growth.

The minimal lethal concentration (MLC) was also determined by inoculating on $\mathrm{MH}$ agar plates $20 \mu \mathrm{L}$ of each concentration tested from 96-well microtiter plates in which no growth was observed. MLC was defined as the lowest concentration that prevented any bacterial growth and reduced the viability of the initial inoculum by at least $99.9 \%$. The tests were carried out in triplicate.

Statistical Analyses. Statistical analysis was performed using different packages (car, HH, agricolae, psych) of the open source statistical program R (version 3.3.3). ${ }^{19}$ All variables were tested for the assumptions of normality and homoscedasticity. A Kruskal-Wallis test applying Bonferroni correction was utilized to compare the results between samples. $p<0.05$ was considered to be significant. In addition, Spearman's correlation coefficient in bivariate linear correlations was used to study the relationship between bioactive compounds and bioactivity.

\section{RESULTS AND DISCUSSION}

Bioactive Compound Quantification (Vitamin C, TPC and TFC). The main bioactive compounds were quantified in the different honey samples. Vitamin $\mathrm{C}$ in honey comes essentially from nectar or honeydew and pollen, whereas phenolic compounds come from propolis in addition to nectar and pollen. ${ }^{20}$ Thus, depending on honey botanical and geographical sources, bioactive compounds content might fluctuate considerably, as hereby reported (Table 3).

Vitamin C was detected in all honey samples. However, contents deeply differed among them, ranging from 0.34 to $75.9 \mathrm{mg} / 100 \mathrm{~g}$ of honey $(p<0.001)$. Sample H7a, a thyme honey, registered significantly higher values of AA, which corroborates that described in previous studies for this variety of honey. ${ }^{21}$ On the other hand, two eucalyptus (H6 and H6a) and two polyfloral honey samples ( $\mathrm{H} 4 \mathrm{a}$ and $\mathrm{H} 7$ ) showed the lowest contents.

Similarly, the amounts of TPC and TFC varied considerably among samples $(p<0.001)$. TPC ranged between 23.1 and $158 \mathrm{mg} / 100 \mathrm{~g}$ of honey and TFC between 1.65 and $5.93 \mathrm{mg} /$ $100 \mathrm{~g}$ of honey. $\mathrm{H1}$, a polyfloral honey, presented the highest values for both TPC and TFC, but in not all cases were the two parameters correlated. The lowest values of TPC and TFC were found in eucalyptus honey (H6 and H6a).

In addition, it is important to take into account that although the Folin-Ciocalteu assay is widely used to determine TPC in food extracts, it is not specific for phenolic quantification, considering that other types of compounds present in honey such as reducing sugars and amino acids can also reduce the Folin-Ciocalteu reagent. ${ }^{22}$ In the present study a correction factor for interfering substances in the determination of TPC was not used because sugars, as principal interaction components in honey, present low solubility in methanol. ${ }^{23}$ Nevertheless, it is necessary to consider that TPC determined may have values higher than the real ones. Similar circumstances occur with TFC; results may show an overestimation as some nonflavonoid compounds can exhibit absorbance at $510 \mathrm{~nm} .{ }^{22}$ However, despite the limitations posed, these methods allow a rapid and estimated evaluation of the availability of these compounds and their potential antioxidant activity. ${ }^{24}$

Identification and Quantification of Individual Polyphenols. Characterization of phenolic compounds and other bioactive components in honey intended for medical uses is essential, since these minor substances might be responsible for many of their health protective effects. ${ }^{3}$

The HPLC-ESI/MS analysis of honey extracts permitted identifcation of 49 different phenolic compounds on the basis of their UV and mass spectra and their MS/MS fragmentations (Table 4). However, only 46 of these were quantified due to some compounds coeluting under a single chromatographic peak with the same retention time (Table 5).

Cinnamic acids and their derivatives were the main phenolic acids found. Three compounds (UI 1, UI 2, and UI 3) were considered unknown but were tentatively identified. UI 3 (UV spectrum 319 sh, 309, $298 \mathrm{sh} n m$; MS $m / z$ 144; $\mathrm{MS}^{2} \mathrm{~m} / z$ 133) was previously described by Tomás-Barberán, Martos, Ferreres, Radovic, and Anklam ${ }^{25}$ as marker of chestnut honey. UI 1 (UV spectrum $328 \mathrm{sh}, 316,306 \mathrm{sh} \mathrm{nm}$; MS m/ $z 188 ; \mathrm{MS}^{2} \mathrm{~m} / z$ 144) and UI 2 (UV spectrum 330, $318 \mathrm{sh} \mathrm{nm;}$ MS $m / z \quad 188 ; M^{2} m / z$ 144) compounds are probably kynurenic acid derivatives in view of the similarities among the UV spectra and MS fragmentations of the three compounds (Table 4). Interestingly, the samples in which Castanea sativa was the predominant or secondary pollen (samples $\mathrm{H} 2, \mathrm{H} 2 \mathrm{a}, \mathrm{H} 5, \mathrm{H} 5 \mathrm{a}, \mathrm{H} 8$, and $\mathrm{H} 8 \mathrm{a}$ ), presented higher amounts of UI 1, UI 2, and UI 3, as well as kynurenic acid, which suggests the relationship between these compounds and a chestnut source. Furthermore, both isomers of abscisic acid previously described in other varieties of honey ${ }^{26}$ were detected but only cis,trans-abscisic acid could be quantified in some samples. Other phenolic compounds, characteristic of $\mathrm{MkH}$, as well as ellagic acid were identified.

Concerning flavonoids, four subclasses of compounds were discriminated: flavonols, flavanonols, flavanones, and flavones, as well as some flavonol glycosides mainly from quercetin, kaempferol, isorhamnetin, and 8-methoxykaempferol, which were previously described in different types of honey. ${ }^{15}$ Moreover, specific floral markers were found in monofloral samples: myricetin and tricetin in eucalyptus honey, ${ }^{15}$ kaempferol and derivatives in rosemary honey, ${ }^{25}$ kynurenic acid in chestnut honey, ${ }^{27}$ and leptosperin, 4-methoxyphenyllactic acid, and methyl syringate in $\mathrm{MkH}^{28}$

The wide variability of honey samples was reflected in the phenolic profiles (Table 5). MkH was very different from the rest, and among other honey samples only eight compounds (quercetin, kaempferol, rhamnetin, quercetin 3,7-dimethyl ether, galangin, pinobanksin, pinocembrin, and chrysin) were common to all of them, as could be expected from their propolis origin and presence in beeswax. Furthermore, results evidenced three types of honey samples: those characterized by profiles dominated by phenolic acids ( $\mathrm{H} 1, \mathrm{H} 2, \mathrm{H} 2 \mathrm{a}, \mathrm{H} 8, \mathrm{H} 8 \mathrm{a}$, and $\mathrm{MkH}$ in which phenolic acids represent between 60.1 and 92.6\% of total phenolic compounds quantified), others in which flavonoids prevailed $(\mathrm{H} 3, \mathrm{H} 4, \mathrm{H} 4 \mathrm{a}, \mathrm{H} 7$, and $\mathrm{H7a}$, in which flavonoids represent between 67.6 and $97.3 \%$ of total phenolic compounds quantified), and finally, those in which none of these compounds stood out (H1a, H5, H5a, H6, and H6a, in which phenolic acids and flavonoids represent around $50 \%$ of total phenolic compounds quantified). 


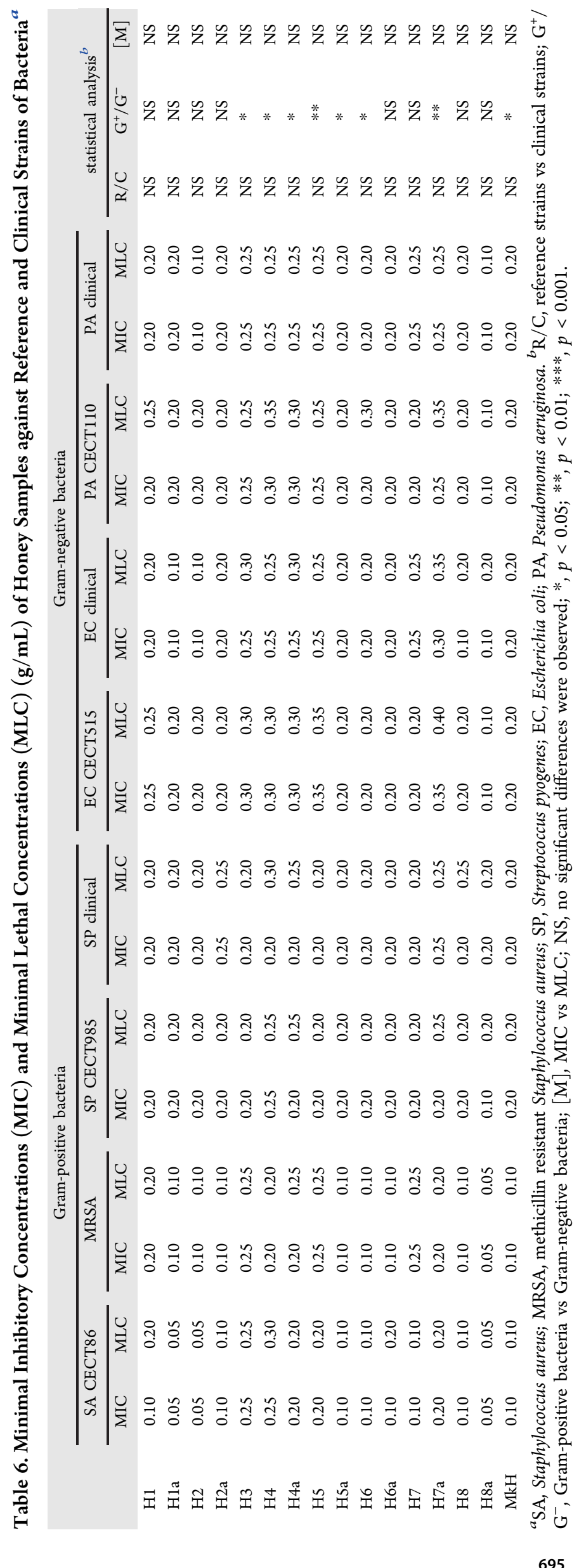


The content of total phenolic compounds ranged between 1.06 and $18.6 \mathrm{mg} / 100 \mathrm{~g}$ of honey in $\mathrm{H7a}$ and $\mathrm{MkH}$, respectively. No correlation between total content of phenolic compounds quantified by HPLC and Folin-Ciocalteu assay was observed. This disparity might be explained because not all phenolic compounds present in honey samples were identified and/or quantified by HPLC and quantification of TPC through a Folin-Ciocalteu assay is only an estimation which was probably overvalued. ${ }^{29}$

In addition to their antioxidant and free radical scavenging abilities, polyphenols possess anti-inflammation, modulation of signal transduction, antimicrobial, and antiproliferation activities. ${ }^{30}$ In addition to quantity, the specific phenolic profile may be a key factor, as particular polyphenols could function individually or act synergistically with other components to increase bioactive properties. ${ }^{10}$ This standpoint highlights the importance of understanding the polyphenol composition of honey samples intended for medical uses.

Antioxidant Activity. Owing to the complex nature of matrix and involvement of multiple reaction characteristics and mechanisms, the antioxidant capacity of honey cannot be evaluated accurately by any single method. Therefore, a combination of assays will provide more information on the antioxidant properties. $^{31,32}$ In the current study three spectrophotometric methods were used.

Regarding the RSA assay, the sample $\mathrm{H} 7 \mathrm{a}$ displayed the lowest concentration able to scavenger $50 \%$ of the free radicals (Table 3). The high concentration of vitamin $\mathrm{C}$ detected in this honey sample could explain the greater activity observed. AA has been described as a reducing agent capable of rapidly catching several reactive oxygen and nitrogen species (ROS and RNS). ${ }^{30,33}$ However, no correlation between AA and RSA was observed. The absence of linear relations between variables does not exclude the presence of other nonlinear associations. Moreover, considering phenolic quantification by HPLC, H7a was the sample with the lowest concentration, which suggests that vitamin $\mathrm{C}$ is responsible for the antioxidant effects.

Similarly, phenolic compounds (TPC and TFC) may elucidate the results regarding the RP assay. On their behalf, phenolic compounds are capable of scavenging free radicals through electron and proton transfer mechanisms, as much as chelating metals, ${ }^{30}$ which could explain the significant correlation observed between TPC and TFC with honey reducing capacity $(R=-0.80,-0.64 ; p<0.01$, respectively). $\mathrm{H} 1$ was the sample that exhibited the highest values of TPC and TFC and likewise the best antioxidant activity in this assay.

Conversely, in the $\beta$-carotene inhibition bleaching assay no correlation was observed with bioactive compounds. The difficulties in finding relationships between data may be due to the lipid/water matrix used, especially because of the emulsifier introduced in the system against phase separation. The emulsifier may change the antioxidant distribution in the emulsified medium, and in turn the antioxidant activity, making it more difficult to interpret the results. Moreover, emulsifiers form micelles, which may trap antioxidants in these self-assembled structures and carry them to the water phase. ${ }^{34}$

In this assay, samples $\mathrm{H} 2 \mathrm{a}, \mathrm{H} 5$, and $\mathrm{H} 5$ a presented similarly high antioxidant activities (more than 90\% inhibition). In contrast, the H7a sample, which presented the best results in the RSA assay, acted as a pro-oxidant. This performance is apparently due to the high content of AA detected in this sample, which indeed exhibited a negative correlation with the inhibition of $\beta$-carotene bleaching $(R=-0.61 ; p<0.05)$. The pro-oxidant behavior of AA has been previously described ${ }^{30,35}$ as a result of the formation of an ascorbyl radical during the oxidation reaction. ${ }^{35}$

A correlation between TPC and antioxidant activity was observed, suggesting that phenolic compounds are some of the main species responsible for the antioxidant capacity of honey. ${ }^{33}$ However, for some samples, similar contents in TPC and TFC did not correspond to similar antioxidant capacities. This suggests that, although phenols remain the largest class of antioxidants found in nature, the overall antioxidant capacity of each sample results from the combined activity of other nonphenolic compounds. ${ }^{32}$

Among those compounds are proteins, amino acids, peptide inhibitors of oxidative enzymes, enzymes such as catalase or/ and glucose oxidase, and organic acids such as gluconic, citric, and malic that could act by chelating metals and thus favor the action of other antioxidants such as polyphenols. ${ }^{11,29}$ Moreover, the antioxidant properties of melanoidins (highmolecular-weight polymers formed in the final stage of the Maillard reaction $)^{36}$ have been described. Finally, because of the complex composition of honey, the interactions among the different compounds with antioxidant capacity and the possible synergies between them can also play an important role in the overall antioxidant capacity. ${ }^{29,31,37}$

Different assays provided different results, since each test assessed diverse action mechanisms in which a great variety of phytochemicals take part.

Antibacterial Activity. Honey antibacterial activity is associated with its physicochemical properties, as much as multiple compounds originating from the nectar of plants, pollen, propolis, and from the honeybee itself. ${ }^{38}$ All honey samples exhibited antibacterial capacity against reference and clinical strains. However, effective concentrations ranged between 0.05 and $0.40 \mathrm{~g} / \mathrm{mL}$ depending on honey variety and microorganism (Table 6).

Overall, $S$. aureus strains seemed to be the most sensitive bacteria, whereas E. coli strains were the most resistant. The outer membrane surrounding the peptidoglycan layer of Gramnegative bacteria offers a greater resistance to the entrance of antimicrobials. $^{31,39}$ However, in the current study, significant differences between Gram-positive and Gram-negative bacteria were not observed for all samples. Being a water-soluble substance, is feasible that honey was capable of accessing the periplasmic space of the bacteria through the porins, which act as hydrophilic conduits, as happens with other water-soluble molecules such as lactic acid. ${ }^{3}$

Significant differences between clinical and reference strains were not observed $(p>0.05)$, suggesting that honey samples were effective even against drug-resistant bacteria. New therapeutic options against emerging multi-drug-resistant pathogens are necessary, even more considering that some common infections have recently become extremely difficult or even impossible to treat. ${ }^{40}$ Due to its peculiarities, honey might be a good option, ${ }^{20,31}$ with little chance to resistance development by acting in a multifactorial way upon several bacteria target sites. ${ }^{41}$ However, this natural substance remains underestimated in mainstream healthcare, in part due to the lack of comprehensive scientific evidence supporting its clinical use. $^{2}$

Furthermore, honey samples exhibited not only bacteriostatic but also bactericidal effects. MLC values were similar or slightly higher than MIC values, and no significant differences between the concentrations were observed $(p>0.05)$. 
Honey antimicrobial activity has been related to physicochemical properties such as high osmolarity, low water activity, and acidity. Moreover, recent studies revealed that polyphenols are key components on antimicrobial effects of honey, ${ }^{10,32}$ on their own or by reacting with $\mathrm{H}_{2} \mathrm{O}_{2}$. Thereby, benzoic acid can react with $\mathrm{H}_{2} \mathrm{O}_{2}$, resulting in peroxy acids, which are more stable and powerful than hydrogen peroxide and are capable of producing bacteria DNA degradation., ${ }^{4,6}$ Conversely, in the present study no significant correlations between phenolic compounds and antibacterial activity were observed, as has been described in other studies. ${ }^{42,43}$ Honey compounds interact among themselves, displaying an additive, synergistic, or antagonistic activity ${ }^{7}$ which might not be explained by a simple linear relation.

To sum up, considering antioxidant activity, the honey samples with greatest potential were $\mathrm{H} 1$ and $\mathrm{H} 2 \mathrm{a}$, corresponding to a polyfloral and a chestnut honey, respectively. However, when the antibacterial capacity was analyzed, the best samples were $\mathrm{H} 1 \mathrm{a}, \mathrm{H} 2$, and $\mathrm{H} 8 \mathrm{a}$, corresponding to an avocado, a chestnut, and a polyfloral honey, respectively. Nevertheless, bioactivity needs to be understood as a combination of beneficial effects, and from this standpoint, $\mathrm{H} 1 \mathrm{a}, \mathrm{H} 2$, and $\mathrm{H} 8 \mathrm{a}$ were the best samples; in addition to a greater antibacterial capacity, their antioxidant potential was appropriate. Although $\mathrm{MkH}$ bioactivity is well-known, in this study other varieties of honey were demonstrated to possess greater activity. Curiously, the phenolic profile seems to be a key factor, since honey samples with greater activity were not related with higher phenolic contents by HPLC, as occurred with H1a. No specific phenolic compounds have been described in avocado honey. Nevertheless, evidence encourages the study of possible markers characteristic of this variety, which could explain its higher bioactive functions.

Moreover, it is essential to underline that although polyfloral honey exhibited good bioactivity, its composition is even more variable than monofloral honeys due to the contribution, in different proportions, of several floral origins without any of them predominating. Not only the major but also a secondary floral source might considerably affect the composition and, consequently, bioactive properties.

Finally, considering that the potential therapeutic application of honey might result in dilution depending on the malady to treat, in vivo concentrations must be greater than those obtained as optimal in vitro, in order to maintain high levels of bioactive compounds in the lesion environment. For some drugs, cytotoxicity may then become a limitation, but this should not be an issue with honey, which could be used undiluted. Defining a correct posology for honey application will be essential for clinical success.

In conclusion, bioactive component contents and related bioactive activities among distinct varieties of honey were rather variable and depended primarily on their botanical origin, which confirms the initial hypothesis. The great variability observed reinforces the necessity to choose a proper type of honey for clinical application. Therefore, screening of a particular honey type composition, as well as its antioxidant and antimicrobial properties, is necessary prior to studies assessing in vivo the therapeutic potential of this natural product.

TPC and TFC provide a rapid and cheap estimation of phenolic compounds present in honey and their potential biological activity. However, these methods could overestimate phenolic content when other interference substances are present; therefore, other techniques that are more precise, such as HPLC-MS, are mandatory. In addition, knowing the phenolic profile is essential in order to identify the association between specific phenolic compounds and particular bioactivity properties.

\section{ASSOCIATED CONTENT}

\section{Supporting Information}

The Supporting Information is available free of charge on the ACS Publications website at DOI: 10.1021/acs.jafc.8b05436.

MIC values for different drugs against the bacteria tested (PDF)

\section{AUTHOR INFORMATION}

\section{Corresponding Author}

*J.M.F.-B.: e-mail, jmfreb@unileon.es; tel, +0034 987291850. ORCID

Francisco A. Tomás-Barberán: 0000-0002-0790-1739

José M. Fresno-Baro: 0000-0002-3438-0852

\section{Funding}

This work was supported by the Consejeria de Sanidad of Junta de Castilla y León (Spain); under grant GRS 551/A/10. P.C.-F. was funded by the Consejeria de Educación of Junta de Castilla y León and European Social Fund.

\section{Notes}

The authors declare no competing financial interest.

\section{ACKNOWLEDGMENTS}

The authors are grateful to Isabel Fernández Natal (Chief of the Clinical Microbiology Service in the University Assistance Complex of León, Spain) for providing us the clinical strains used in this work and drug susceptibility information.

\section{REFERENCES}

(1) Eteraf-Oskouei, T.; Najafi, M. Traditional and Modern Uses of Natural Honey in Human Diseases: A Review. Iran. J. Basic Med. Sci. 2013, 16 (6), 731-742.

(2) Carter, D. A.; Blair, S. E.; Cokcetin, N. N.; Bouzo, D.; Brooks, P.; Schothauer, R.; Harry, E. J. Therapeutic Manuka Honey: No Longer So Alternative. Front. Microbiol. 2016, 7, 569.

(3) Alvarez-Suarez, J. M.; Tulipani, S.; Díaz, D.; Estevez, Y.; Romandini, S.; Giampieri, F.; Damiani, E.; Astolfi, P.; Bompadre, S.; Battino, M. Antioxidant and Antimicrobial Capacity of Several Monofloral Cuban Honeys and Their Correlation with Color, Polyphenol Content and Other Chemical Compounds. Food Chem. Toxicol. 2010, 48 (8-9), 2490-2499.

(4) Oryan, A.; Alemzadeh, E.; Moshiri, A. Biological Properties and Therapeutic Activities of Honey in Wound Healing: A Narrative Review and Meta-Analysis. J. Tissue Viability 2016, 25, 98-118.

(5) Al-Waili, N. S.; Salom, K.; Butler, G.; Al Ghamdi, A. A. Honey and Microbial Infections: A Review Supporting the Use of Honey for Microbial Control. J. Med. Food 2011, 14 (10), 1079-1096.

(6) Brudzynski, K.; Abubaker, K.; Miotto, D. Unraveling a Mechanism of Honey Antibacterial Action: Polyphenol/H2O2Induced Oxidative Effect on Bacterial Cell Growth and on DNA Degradation. Food Chem. 2012, 133 (2), 329-336.

(7) Kwakman, P. H. S.; Zaat, S. A. J. Antibacterial Components of Honey. IUBMB Life 2012, 64 (1), 48-55.

(8) Bong, J.; Loomes, K. M.; Lin, B.; Stephens, J. M. New Approach: Chemical and Fluorescence Profiling of NZ Honeys. Food Chem. 2018, 267, 355-367.

(9) Lin, B.; Loomes, K. M.; Prijic, G.; Schlothauer, R.; Stephens, J. M. Lepteridine as a Unique Fluorescent Marker for the Authentication of Manuka Honey. Food Chem. 2017, 225, 175-180. 
(10) Fyfe, L.; Okoro, P.; Paterson, E.; Coyle, S.; McDougall, G. J. Compositional Analysis of Scottish Honeys with Antimicrobial Activity against Antibiotic-Resistant Bacteria Reveals Novel Antimicrobial Components. LWT - Food Sci. Technol. 2017, 79, 52-59.

(11) Hossen, M. S.; Ali, M. Y.; Jahurul, M. H. A.; Abdel-Daim, M. M.; Gan, S. H.; Khalil, M. I. Beneficial Roles of Honey Polyphenols against Some Human Degenerative Diseases: A Review. Pharmacol. Rep. 2017, 69 (6), 1194-1205.

(12) Combarros-Fuertes, P.; Valencia-Barrera, R. M.; Estevinho, L. M.; Dias, L. G.; Castro, J. M.; Tornadijo, M. E.; Fresno, J. M. Spanish Honeys with Quality Brand: A Multivariate Approach to Physicochemical Parameters Microbiological Quality and Floral Origin. J. Apic. Res. 2018, 1.

(13) AOAC. Official Method 967.21. Ascorbic Acid in Vitamin Preparations and Juices, 18th ed.; AOAC International: Gaithersburg, MD, USA, 2006.

(14) Silici, S.; Sagdic, O.; Ekici, L. Total Phenolic Content, Antiradical, Antioxidant and Antimicrobial Activities of Rhododendron Honeys. Food Chem. 2010, 121 (1), 238-243.

(15) Truchado, P.; Ferreres, F.; Tomas-Barberan, F. A. Liquid Chromatography-tandem Mass Spectrometry Reveals the Widespread Occurrence of Flavonoid Glycosides in Honey, and Their Potential as Floral Origin Markers. Adv. Sep. Methods Food Anal. 2009, 1216 (43), 7241-7248.

(16) Ferreira, I. C. F. R.; Aires, E.; Barreira, J.; Estevinho, L. M. Antioxidant Activity of Portuguese Honey Samples: Different Contributions of the Entire Honey and Phenolic Extract. Food Chem. 2009, 114 (4), 1438-1443.

(17) Guerrini, A.; Bruni, R.; Maietti, S.; Poli, F.; Rossi, D.; Paganetto, G.; Muzzoli, M.; Scalvenzi, L.; Sacchetti, G. Ecuadorian Stingless Bee (Meliponinae) Honey: A Chemical and Functional Profile of an Ancient Health Product. Food Chem. 2009, 114 (4), $1413-1420$.

(18) CLSI. Methods for Dilution Antimicrobial Susceptibility Tests for Bacteria That Grow Aerobically; Approved Standard, 9th ed.; CLSI Document M07-A9; Wayne, PA, 2012.

(19) R Core Team. R: A Language and Environment for Statistical Computing; R Foundation for Statistical Computing: Vienna, Austria, 2016.

(20) Da Silva, I. A. A.; Da Silva, T. M. S.; Camara, C. A.; Queiroz, N.; Magnani, M.; de Novais, J. S.; Soledade, L. E. B.; Lima, E. de O.; de Souza, A. L.; de Souza, A. G. Phenolic Profile, Antioxidant Activity and Palynological Analysis of Stingless Bee Honey from Amazonas, Northern Brazil. Food Chem. 2013, 141 (4), 3552-3558.

(21) León-Ruiz, V.; Vera, S.; González-Porto, A. V.; San Andrés, M. P. Vitamin C and Sugar Levels as Simple Markers for Discriminating Spanish Honey Sources. J. Food Sci. 2011, 76 (3), C356-C361.

(22) Silva, K. D. R. R.; Sirasa, M. S. F. Antioxidant Properties of Selected Fruit Cultivars Grown in Sri Lanka. Food Chem. 2018, 238, 203-208.

(23) Montañés, F.; Olano, A.; Ibáñez, E.; Fornari, T. Modeling Solubilities of Sugars in Alcohols Based on Original Experimental Data. AIChE J. 2007, 53 (9), 2411-2418.

(24) Nguyen, H. T. L.; Panyoyai, N.; Paramita, V. D.; Mantri, N.; Kasapis, S. Physicochemical and Viscoelastic Properties of Honey from Medicinal Plants. Food Chem. 2018, 241, 143-149.

(25) Tomás-Barberán, F. A.; Martos, I.; Ferreres, F.; Radovic, B. S.; Anklam, E. HPLC Flavonoid Profiles as Markers for the Botanical Origin of European Unifloral Honeys. J. Sci. Food Agric. 2001, 81 (5), $485-496$.

(26) Ferreres, F.; Andrade, P.; Tomás-Barberán, F. A. Natural Occurrence of Abscisic Acid in Heather Honey and Floral Nectar. J. Agric. Food Chem. 1996, 44 (8), 2053-2056.

(27) Truchado, P.; Martos, I.; Bortolotti, L.; Sabatini, A. G.; Ferreres, F.; Tomas-Barberan, F. A. Use of Quinoline Alkaloids as Markers of the Floral Origin of Chestnut Honey. J. Agric. Food Chem. 2009, 57 (13), 5680-5686.

(28) Kato, Y.; Fujinaka, R.; Ishisaka, A.; Nitta, Y.; Kitamoto, N.; Takimoto, Y. Plausible Authentication of Manuka Honey and Related
Products by Measuring Leptosperin with Methyl Syringate. J. Agric. Food Chem. 2014, 62 (27), 6400-6407.

(29) Gheldof, N.; Engeseth, N. J. Antioxidant Capacity of Honeys from Various Floral Sources Based on the Determination of Oxygen Radical Absorbance Capacity and Inhibition of in Vitro Lipoprotein Oxidation in Human Serum Samples. J. Agric. Food Chem. 2002, 50 (10), 3050-3055.

(30) Oroian, M.; Escriche, I. Antioxidants: Characterization, Natural Sources, Extraction and Analysis. Food Res. Int. 2015, 74, 10-36.

(31) Tenore, G. C.; Ritieni, A.; Campiglia, P.; Novellino, E. Nutraceutical Potential of Monofloral Honeys Produced by the Sicilian Black Honeybees (Apis Mellifera Ssp. Sicula). Food Chem. Toxicol. 2012, 50 (6), 1955-1961.

(32) Sousa, J. M.; de Souza, E. L.; Marques, G.; Meireles, B.; de Magalhães Cordeiro, Â. T.; Gullón, B.; Pintado, M. M.; Magnani, M. Polyphenolic Profile and Antioxidant and Antibacterial Activities of Monofloral Honeys Produced by Meliponini in the Brazilian Semiarid Region. Food Res. Int. 2016, 84, 61-68.

(33) Perna, A.; Intaglietta, I.; Simonetti, A.; Gambacorta, E. A Comparative Study on Phenolic Profile, Vitamin C Content and Antioxidant Activity of Italian Honeys of Different Botanical Origin. Int. J. Food Sci. Technol. 2013, 48 (9), 1899-1908.

(34) Shahidi, F.; Zhong, Y. Measurement of Antioxidant Activity. J. Funct. Foods 2015, 18, 757-781.

(35) Hassimotto, N. M. A.; Genovese, M. I.; Lajolo, F. M. Antioxidant Activity of Dietary Fruits, Vegetables, and Commercial Frozen Fruit Pulps. J. Agric. Food Chem. 2005, 53 (8), 2928-2935.

(36) Brudzynski, K.; Miotto, D. Honey Melanoidins: Analysis of the Compositions of the High Molecular Weight Melanoidins Exhibiting Radical-Scavenging Activity. Food Chem. 2011, 127 (3), 1023-1030.

(37) Escuredo, O.; Míguez, M.; Fernández-González, M.; Carmen Seijo, M. Nutritional Value and Antioxidant Activity of Honeys Produced in a European Atlantic Area. Food Chem. 2013, 138 (2-3), $851-856$.

(38) Salonen, A.; Virjamo, V.; Tammela, P.; Fauch, L.; JulkunenTiitto, R. Screening Bioactivity and Bioactive Constituents of Nordic Unifloral Honeys. Food Chem. 2017, 237, 214-224.

(39) Alakomi, H. L.; Skyttä, E.; Saarela, M.; Mattila-Sandholm, T.; Latva-Kala, K.; Helander, I. M. Lactic Acid Permeabilizes GramNegative Bacteria by Disrupting the Outer Membrane. Appl. Environ. Microbiol. 2000, 66 (5), 2001-2005.

(40) Prestinaci, F.; Pezzotti, P.; Pantosti, A. Antimicrobial Resistance: A Global Multifaceted Phenomenon. Pathog. Global Health 2015, 109 (7), 309-318.

(41) Cooper, R. A.; Jenkins, L.; Henriques, A. F. M.; Duggan, R. S.; Burton, N. F. Absence of Bacterial Resistance to Medical-Grade Manuka Honey. Eur. J. Clin. Microbiol. Infect. Dis. 2010, 29 (10), 1237-1241.

(42) Isidorov, V. A.; Bagan, R.; Bakier, S.; Swiecicka, I. Chemical Composition and Antimicrobial Activity of Polish Herbhoneys. Food Chem. 2015, 171, 84-88.

(43) Lukasiewicz, M.; Kowalski, S.; Makarewicz, M. Antimicrobial an Antioxidant Activity of Selected Polish Herbhoneys. LWT - Food Sci. Technol. 2015, 64 (2), 547-553. 